\title{
Engineered Exosomes for the Targeted Delivery of a Novel Therapeutic Cargo to Enhance Sorafenib- Mediated Ferroptosis in Hepatocellular Carcinoma.
}

\section{Xiaoju Li}

Fourth Military Medical University: Air Force Medical University

Qianqian Yu

Huazhong University of Science and Technology Tongji Medical College

\section{Xinyan Guo}

Fourth Military Medical University: Air Force Medical University

Chenlin Liu

Fourth Military Medical University: Air Force Medical University

Runze Zhao

Fourth Military Medical University: Air Force Medical University

Kuo Zhang

Fourth Military Medical University: Air Force Medical University

\section{Wangqian Zhang}

Fourth Military Medical University: Air Force Medical University

Jinghan Liu

Fourth Military Medical University: Air Force Medical University

\section{Guanchao Sun}

Fourth Military Medical University: Air Force Medical University

\section{Shuning Wang}

Fourth Military Medical University: Air Force Medical University

Qiang Hao

Fourth Military Medical University: Air Force Medical University

\section{Weina Li}

Fourth Military Medical University: Air Force Medical University

\section{Meng Li}

Fourth Military Medical University: Air Force Medical University

\section{Yingqi Zhang}

Fourth Military Medical University: Air Force Medical University

\section{Cun Zhang}

Fourth Military Medical University: Air Force Medical University

\section{Yuan Gao ( $\nabla$ gaooyuan@hku.hk)}




\section{Research Article}

Keywords: hepatocellular carcinoma, sorafenib, engineered exosome, ferroptosis

Posted Date: December 1st, 2021

DOI: https://doi.org/10.21203/rs.3.rs-1108956/v1

License: (c) (1) This work is licensed under a Creative Commons Attribution 4.0 International License. Read Full License 


\section{Abstract \\ Background}

Sorafenib is one of the few effective first-line drugs approved for the treatment of advanced hepatocellular carcinoma (HCC). However, the development of drug resistance is common among individuals with HCC. Thus, there is an urgent need to solve this problem.

\section{Results}

Recent evidence indicated that the anticancer activity of sorafenib mainly relies on the induction of ferroptosis. In our study, genes that suppress ferroptosis, especially GPX4 and DHODH, were enriched in sorafenib-resistant cells and primary tissues and were associated with poor prognosis of HCC patients who received sorafenib treatment. Therefore, silencing GPX4 and DHODH might be a novel and effective strategy to overcome sorafenib resistance. Here, a novel ferroptosis inducer comprising a multiplex small interfering RNA (multi-siRNA) capable of simultaneously silencing GPX4 and DHODH was created. Then, exosomes with high multi-siRNA loading and HCC-specific targeting were established by fusing the SP94 peptide and the N-terminal RNA recognition motif (RRM) of U1-A with the exosomal membrane protein Lamp2b. The results from the in vitro and in vivo experiments indicate that this tumor-targeting

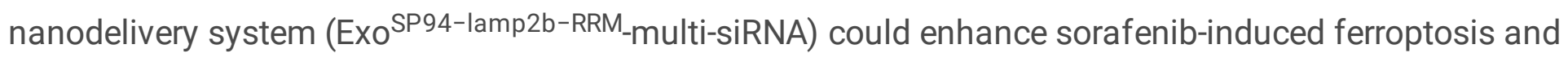
overcome sorafenib resistance, which might open a new avenue for clinically overcoming sorafenib resistance.

\section{Conclusions}

We designed HCC-targeted exosomes (Exo ${ }^{\text {SP94-Lamp2b-RRM) }}$ that can deliver a novel ferroptosis inducer.

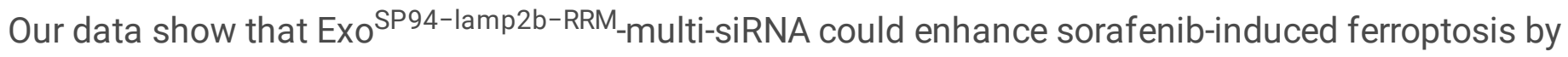
silencing GPX4 and DHODH expression and consequently increase HCC sensitivity to sorafenib. This is the first study to describe the use of engineered exosomes to overcome acquired sorafenib resistance with respect to ferroptosis.

\section{Background}

Hepatocellular carcinoma (HCC) was ranked as the sixth most commonly diagnosed cancer and the fourth leading cause of cancer-related death worldwide in 2018[1, 2]. In the early stages of HCC, curative treatment can be achieved with tumor ablation, resection, or liver transplantation[3]. However, the majority of HCC patients have middle- or late-stage disease at the time of diagnosis, which is past the optimal window for curative treatment. Sorafenib is the first systemic therapy shown to improve survival in HCC and has been approved by the U.S. Food and Drug Administration (FDA) for treatment of unresectable HCC[4]. Despite their initial response, sorafenib-treated tumors rarely regress completely, and most 
patients develop disease progression. Therefore, to improve the survival and quality of life of patients with HCC, combination therapies should be considered as a potentially superior treatment option.

Ferroptosis is a recently discovered form of programmed cell death characterized by iron-dependent accumulation of lipid peroxides to lethal amounts[5]. A growing amount of evidence indicates that ferroptosis can be induced by inhibiting cystine/glutamate transporter (system $\mathrm{x}_{\mathrm{c}^{-}}$) activity, downregulating GPX4 or DHODH expression, and accumulating reactive oxygen species (ROS)[6]. Recent reports have shown that sorafenib can induce ferroptosis by inhibiting system $\mathrm{x}_{\mathrm{c}}$-[7]. Moreover, numerous studies suggest that the anticancer activity of sorafenib mainly relies on inducing ferroptosis[5, 8-11]. Therefore, targeting constituents of ferroptosis might be a promising strategy to increase sorafenib efficacy and overcome sorafenib resistance.

Herein, we first found that ferroptosis suppressor genes, especially GPX4 and DHODH, are enriched in sorafenib-resistant cells and primary tissues from patients and are associated with poor prognosis of HCC patients who receive sorafenib treatment. Then, we created a novel ferroptosis inducer comprising a multiplex small interfering RNA (multi-siRNA) suitable for simultaneously silencing GPX4 and DHODH. Then, exosomes (Exo ${ }^{\text {SP94-lamp2b-RRM }}$ ) with high tumor targeting ability and high multi-siRNA loading efficacy were constructed to deliver the multi-siRNA cargo. Using in vitro and in vivo models, we demonstrated that this tumor-targeting nanodrug (Exo ${ }^{\text {SP94-lamp2b-RRM }}$-multi-siRNA) could enhance sorafenib-induced ferroptosis and overcome sorafenib resistance, suggesting that it is a promising therapeutic strategy for treating sorafenib-resistant HCC.

\section{Results}

\subsection{Suppressed ferroptotic activity during sorafenib treatment is associated with compromised therapeutic efficiency.}

To confirm whether sorafenib could induce ferroptosis in HCC cells, we performed a CCK-8 assay. Our data showed that sorafenib-mediated cell death in the human HCC cell line HepG-2 was blocked by ferrostatin-1 (a ferroptosis inhibitor) (Figure 1A, Figure S1A). The accumulation of reactive oxygen species (ROS) and lipid peroxidation are key events in ferroptosis[12]; thus, we analyzed the levels of ROS and the end products of lipid peroxidation (i.e., MDA) in HepG-2 cells. The results indicated that sorafenib increased the levels of ROS and MDA in HepG-2 cells (Figure 1B-C). Apart from genes that function as ferroptosis-suppressing agents, numerous genes have been identified as key ferroptosis suppressors. For example, glutathione peroxidase 4 (GPX4) resides in the center of a network that functions to prevent the accumulation of lipid hydroperoxides, thereby strongly suppressing ferroptosis[13]. Interestingly, we constructed a ferroptosis suppressor gene signature and discovered that ferroptosis suppressor genes were enriched in sorafenib-resistant cells and primary tissues from HCC patients (Figure 1D, Figure S1B). Moreover, the results indicated that the high expression level of the ferroptosis suppressor gene signature 
was associated with poor prognosis of HCC patients who received sorafenib treatment (Figure 1E, Figure S1C). Therefore, the results suggest that suppressed ferroptotic activity is associated with sorafenib resistance.

\subsection{Gene-silencing activities of the multi-siRNA against GPX4 and DHODH.}

The above data suggest that silencing key ferroptosis suppressor genes increases the therapeutic effect of sorafenib. Among the ferroptosis suppressor genes, GPX4 and DHODH can directly remove the dangerous products of iron-dependent lipid peroxidation and consequently protect the cell membrane from damage; when GPX4 and DHODH expression and/or function are dysregulated, ferroptosis ensues[14, 15]. Moreover, we found that the expression of GPX4 and DHODH was upregulated in sorafenib-resistant patients (Figure 2A-B) and associated with poor prognosis of HCC patients who received sorafenib treatment (Figure 2C-D). Meanwhile, GPX4 and DHODH expression was upregulated in sorafenib-resistant HCC cells (Figure 2E). In light of these findings, we sought to interfere with the expression and function of these two genes at the same time. First, siRNAs targeting the human GPX4 gene and human DHODH gene were designed and screened to determine their effectiveness. As shown in Figure 2F-G and Figure S2A-B, si-GPX4\#1 and si-DHODH\#1 were the most effective siRNAs in knocking down their respective proteins. Then, we designed a multi-siRNA containing the sequences corresponding to si-GPX4\#1 and si-DHODH\#1; the sequence "AUUGCAC" was used as a linker to connect si-GPX4\#1 to si-DHODH\#1. The results showed that this multi-siRNA could simultaneously suppress GPX4 and DHODH expression (Figure $2 \mathrm{H}$ ).

\subsection{Preparation and characterization of SP94-Lamp2b-RRM fusion protein-engineered exosomes}

Exosomes have been considered a good siRNA delivery vehicle to reduce drug resistance[16]. However, low cargo encapsulation efficiency and the lack of cell-type specific targeting remain major hurdles for their potential clinical application. Recently, some RNA-binding proteins have been shown to significantly promote exosomal miRNA cargo loading via interaction with common short sequence motifs present in miRNAs[17]. Therefore, we hypothesized that fusion of specific RNA-binding proteins with the exosomal membrane proteins can increase the loading efficiency of the desired RNAs. We fused the N-terminal RNA recognition motif (RRM) of U1-A with the C-terminus of Lamp2b (exosomal surface protein) (Figure 3A-B). U1-A can bind the highly conserved sequence "AUUGCAC" in RNA via its N-terminal RRM[18]. SP94 (protein sequence, SFSIIHTPILPL), is a novel peptide that has been reported to specifically bind to HCC cells[19]. Therefore, we fused SP94 with the N-terminus of Lamp2b to enhance the tumor-targeting ability of the engineered exosomes (Figure $3 A-B$ ). The recombinant vector produced abundant expression of the fusion protein in transfected cells (Figure 3C). As the engineered SP94-Lamp2b-RRM fusion protein could express on exosomes (Figure 3D), we analyzed the binding ability of these exosomes to HCC cells. The results showed that exosomes derived from HEK-293T cells transfected with SP94-Lamp2b-RRM could bind strongly to HCC cells (Figure 3E), indicating that the SP94-Lamp2b-RRM fusion protein was 
incorporated into exosomes. In addition, specific exosome markers (CD63, TSG101 and CD9) were detected in the purified samples, while the Golgi marker GM130 was barely detectable (Figure 3F). Nanoparticle tracking analysis (NTA) revealed that the exosomes were similar in number and had a similar size distribution between the groups (Figure 3G). Transmission electron microscopy (TEM) confirmed that the purified exosomes exhibited a typical round-shaped vesicular morphology and were within the appropriate size range (Figure $3 \mathrm{H}$ ).

\subsection{Efficient therapeutic cargo encapsulation into SP94- Lamp2b-RRM fusion-protein-engineered exosomes.}

Next, we explored whether the engineered SP94-Lamp2b-RRM fusion protein could promote RNA loading during exosome biogenesis. First, HEK-293T cells overexpressing the SP94-Lamp2b-RRM fusion protein were transfected with $100 \mathrm{nM}$ corresponding FITC-tagged multi-siRNA (Figure 4A). Then, an equal number of exosomes from each group was evaluated by flow cytometry. The results showed that a higher amount of multi-siRNA containing the RRM recognition motif "AUUGCAC" could be sorted into exosomes compared to that of multi-siRNA\#2 without the RRM binding sequence (Figure 4B). HEK-293T cells were then cocultured with HepG-2 cells (Figure 4C). Images from the confocal microscope showed a higher level of multi-siRNA containing the RRM "AUUGCAC" in HepG-2 cells (Figure 4D). Consistent with this finding, exosomes derived from HEK-293T cells transfected with the multi-siRNA containing the "AUUGCAC" sequence could significantly suppress the expression of GPX4 and DHODH in HCC cells (Figure 4E). These results suggest that the SP94-Lamp2b-RRM fusion protein promotes the exosomal loading of the "AUUGCAC" sequence linked to the multi-siRNA via RNA-protein interactions.

\subsection{SP94-Lamp2b-RRM-functionalized exosomes could overcome sorafenib resistance by enhancing sorafenib- induced ferroptosis in HCC cells.}

To investigate whether Exo ${ }^{\text {SP94-Lamp2b-RRM }}$ can bind to HCC cells, blank exosomes or Exo ${ }^{\text {SP94-Lamp2b-RRM }}$ were labeled with DiR and added to sorafenib-resistant HepG-2 cell cultures (Figure 5A). The results indicated that the introduction of the SP94 targeting peptide dramatically enhanced the binding ability of the exosomes to target the cancer cells (Figure 5B). Then, we treated sorafenib-resistant HepG-2 cells with

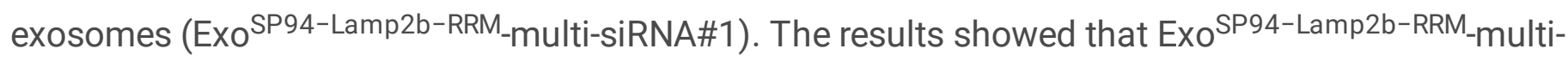
siRNA\#1 could significantly enhance the effect of sorafenib and that this effect was compromised by the ferroptosis inhibitor ferroptosis-1 (Figure $5 \mathrm{C}$ ). Then, we further measured ROS and lipid peroxidation levels, which are the primary drivers of ferroptosis. The results showed that treatment with

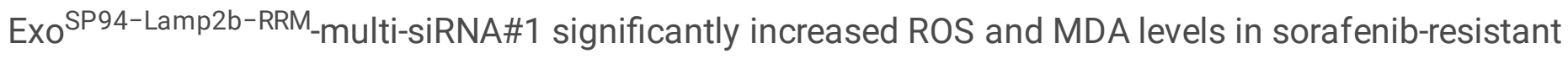
cells (Figure 5D-E). In addition to biochemical analyses, TEM was used to observe morphological

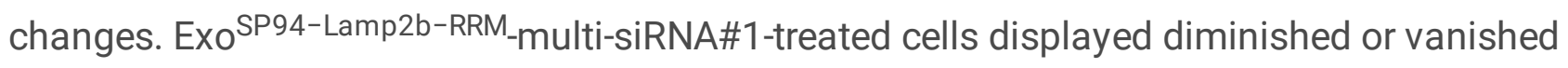
mitochondrial cristae and condensed mitochondrial membrane densities compared to

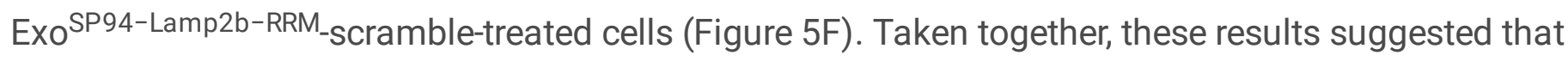




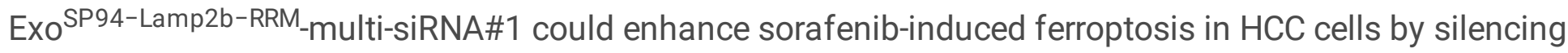
the expression of GPX4 and DHODH.

\subsection{SP94-Lamp2b-RRM-functionalized exosomes could efficiently target hepatocellular carcinoma in vivo.}

To confirm whether SP94-Lamp2b-RRM-functionalized exosomes could target HCC in vivo, we tracked the distribution of DiR-labeled exosomes. First, we established an orthotopic liver injection model and observed that SP94-Lamp2b-RRM-functionalized exosomes were mainly distributed to the liver (Figure 6A-C). Meanwhile, SP94-Lamp2b-RRM-functionalized exosomes could target HCC (Figure 6A-C). Next, sorafenib-resistant HepG-2 cells were subcutaneously inoculated into the left backs of mice. In the subcutaneous HCC model, we found that SP94-Lamp2b-RRM-functionalized exosomes were mainly distributed in the liver and subcutaneous tumor tissues (Figure 6D, Figure S3A). The results from the different HCC models suggest that the SP94 targeting peptide dramatically enhances the ability of exosomes to bind HCC cells and tissues.

\subsection{Therapeutic effects of cotreatment with SP94-Lamp2b- RRM-functionalized exosomes and sorafenib in an HCC mouse model.}

Next, we explored the therapeutic effect of SP94-Lamp2b-RRM-functionalized exosomes combined with sorafenib in vivo. While the tumors of mice treated with sorafenib and Exo ${ }^{\text {SP94-Lamp2b-RRM }}$ containing scramble multi-siRNA grew rapidly, those of mice treated with sorafenib and ExoSP94-Lamp2b-RRM containing multi-siRNA\#1 were significantly reduced after 21 days of treatment (Figure 7A-B). However, the effect of sorafenib and Exo ${ }^{\text {SP94-Lamp2b-RRM }}$ containing multi-siRNA\#1 on inhibiting tumor growth was diminished when cells were treated with the ferroptosis inhibitor ferrostatin-1 (Figure 7A-B). Similarly, mice from the sorafenib and Exo ${ }^{\text {SP94-Lamp2b-RRM }}$ containing multi-siRNA\#1 cotreatment group had a longer life expectancy than did mice from the sorafenib and Exo ${ }^{\text {SP94-Lamp2b-RRM }}$ containing scramble multi-siRNA treatment group (Figure 7C). To examine the knockdown efficiency in each group, GPX4 and DHODH expression in primary tumor lesions was investigated by immunohistochemistry. The results showed that multi-siRNA\#1 could significantly suppress GPX4 and DHODH expression in vivo (Figure

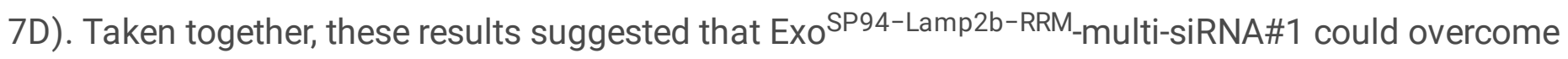
sorafenib resistance in vivo by silencing the ferroptosis suppressor genes GPX4 and DHODH.

\subsection{Systemic toxicity evaluation}

To evaluate the systemic toxicity of Exo ${ }^{\text {SP94-Lamp2b-RRM }}$, exosomes containing scramble or multi-siRNA\#1 were injected into nude mice via tail vein. In addition, there was no significant difference in the body weights of mice in the two groups (Figure S4A). In addition, compared to the control group, the Exo ${ }^{\text {SP94-Lamp2b-RRM }}$ group showed relatively normal tissue structure and morphology (Figure 8A). These results indicate that there was no obvious adverse effect after treatment with Exo SP94-Lamp2b-RRM. 


\section{Discussion}

Our study demonstrated that sorafenib could induce ferroptosis in HCC cells, which is in line with the literature stating that the anticancer activity of sorafenib mainly relies on the induction of ferroptosis. However, we found that the expression of ferroptosis suppressor genes, especially GPX4 and DHODH, was enriched in sorafenib-resistant HCC cells and patient samples, which suggests that suppressed ferroptotic activity is associated with compromised therapeutic efficiency of sorafenib. Thus, precise elimination of ferroptosis suppressor genes might be a new promising strategy to enhance sorafenib efficacy and improve patient prognosis.

Ferroptosis is a form of regulated cell death that is induced by excessive lipid peroxide accumulation in cellular membranes[20-22]. Cells express two genes that function as defensive mechanisms to safely process lipid peroxides: GPX4 and DHODH. Consequently, disabling one arm forces cells to be more dependent on the other. For example, inhibition of either DHODH or GPX4 alone did not induce ferroptosis in HT-1080 cells, which is likely due to the relatively high endogenous expression of GPX4 and DHODH[23]; interestingly, a GPX4 inhibitor combined with a DHODH inhibitor could synergistically suppress HT-1080 tumor growth. Similarly, in our study, the sorafenib-resistant cells and tissue samples exhibited high levels of GPX4 and DHODH expressed. Therefore, targeting both genes might better enhance sorafenib-induced ferroptosis. Here, we created a multi-siRNA that can simultaneously knock down GPX4 and DHODH in vitro and in vivo. To our knowledge, this is the first ferroptosis inducer that can directly target two genes. Additionally, this novel construct provides a new approach for the clinical treatment of sorafenib-resistant cancer.

Because of their intrinsic nature, exosomes are biocompatible with the host immune system and have an innate ability to protect and transport small RNAs and other critical molecules across biological barriers in vivo; they have been increasingly recognized as promising vehicles to deliver siRNA in vivo[24, 25]. At present, the most common way to load nucleic acids into exosomes is via electroporation or direct encapsulation in donor cells[26]; however, these methods have relatively low loading efficiencies. Meanwhile, in vitro loading of naked siRNAs into exosomes by electroporation could cause extensive siRNA aggregation and significantly reduce the level of bioactive siRNAs[27]. Thus, there is an urgent need to develop a strategy for efficient loading of nucleic acids, especially siRNA. Recently, research conducted by our laboratory and others have observed that some RNA-binding proteins can efficiently sort miRNAs and other RNAs into exosomes via protein-RNA interactions[28, 29]. In light of these data, we hypothesized that fusion of an exosomal membrane protein with a specific RNA-binding protein would increase the loading efficiency of the siRNA of interest; therefore, we fused the RNA recognition motif of U1-A with the C-terminus of Lamp2b, which interacts with the sequence "AUUGCAC" in the target RNA with a relatively high affinity. Then, a multi-siRNA was engineered to harbor the consensus "AUUGCAC" sequence. The preliminary data here indicate that the fusion protein helps sort multiple siRNAs containing the "AUUGCAC" sequence into exosomes during exosome biogenesis, especially when the RNA of interest is exogenously overexpressed. Our study established a novel strategy to efficiently load therapeutic multi-siRNA cargos into exosomes. 
Currently, the poor solubility and potential off-target toxicity to normal cells and tissues preclude the systematic use of traditional ferroptosis inducers in vivo[30-32]. Nanoscale exosomes are considered a good choice as a drug vehicle because exosomes could be engineered toward better targeting specificity via exosome surface protein modifications[33-37]. For example, exosomes expressing the ay integrinspecific iRGD peptide fused to LAMP-2b efficiently delivered doxorubicin to integrin-positive breast cancer cells in vitro and in vivo[38]. Here, an HCC-specific targeting peptide, SP94, was fused to the extracellular domain of LAMP-2B at the N-terminus[19]. Our data show that functionalized exosomes

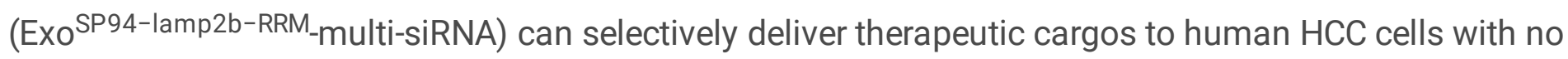
obvious adverse effects. This is the first report of tumor-targeted exosome delivery of ferroptosis inducers, which might open a new avenue for the systematic use of ferroptosis inducers to treat cancer.

In this study, we designed HCC-targeted engineered exosomes (Exo ${ }^{\text {SP94-Lamp2b-RRM) }}$ to deliver a novel

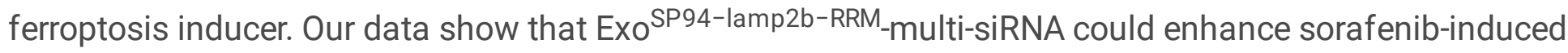
ferroptosis by knocking down GPX4 and DHODH and consequently increase the sensitivity of HCC to sorafenib. This is the first study describing the use of engineered exosomes to overcome acquired resistance to sorafenib from the perspective of ferroptosis.

\section{Methods}

\section{Antibodies and inhibitors}

The antibodies used targeted the following proteins (dilutions used are included):

GAPDH (CW0101: immunoblotting, 1:1000) from CWBIOTECH; F-actin (40734ES75: immunofluorescence, 1:100) from YEASEN; SNRPA (10212: immunoblotting, 1:500) from Proteintech; CD63 (67605-1-lg: immunoblotting, 1:5000) from Proteintech; CD9 (60232-1-Ig: immunoblotting, 1:5000) from Proteintech; TSG101 (14497-1-AP: immunoblotting, 1:1000) from Proteintech; GPX4 (67763-1-lg: immunoblotting, 1:5000; IHC, 1:1000) from Proteintech; and DHODH (14877-1-AP: immunoblotting, 1:2000; IHC, 1:100) from Proteintech.

The inhibitors used are as follows:

Ferrostatin-1 (HY-100579: $60 \mathrm{nM}$ for the in vitro assay) was obtained from MedChem Express, and ferrostatin-1 (HY-100579: $5 \mathrm{mg} / \mathrm{kg}$, intraperitoneal injection for the in vivo assay) was obtained from MedChem Express.

\section{Cell lines and culture}

The HepG-2 and HEK-293T cell lines were obtained from the Type Culture Collection of the Chinese Academy of Sciences (Shanghai, China). These cell lines were authenticated by the analysis of short tandem repeat (STR) profiles, and all of them matched those of the standard cell lines in the DSMZ data bank. These cells tested negative for cross-contamination of other human cells and mycoplasma 
contamination. HepG-2 and HEK-293T cells were cultured in DMEM containing 10\% fetal bovine serum and $1 \%$ penicillin-streptomycin.

\section{Transmission electron microscope assay}

Cells were collected and fixed with $2.5 \%$ glutaraldehyde. Subsequently, cells were postfixed in $2 \%$ tetroxide and dehydrated through a series of gradient ethanol solutions. Samples were embedded in epoxy resin, cut into thin slices, and placed onto a nickel grid. Images were acquired using a Tecnai G2 Spirit transmission electron microscope (Thermo Fisher).

\section{Intracellular ROS measurements}

A lipid ROS assay was performed as described previously. Briefly, cells were incubated with PBS containing $10 \mu \mathrm{M}$ DCFDA dye in a cell culture incubator for $30 \mathrm{~min}$. Cells were then collected and washed twice with PBS followed by resuspension in $200 \mu$ l of PBS. ROS levels were analyzed using a Beckman CytoFLEX system through the FITC channel.

\section{Detection of malondialdehyde (MDA)}

Analysis of lipid peroxidation was assessed by quantifying the MDA concentration in cell lysates using a Lipid Peroxidation MDA Assay Kit (S0131, Beyotime) in accordance with the manufacturer's instructions.

\section{Immunohistochemistry}

This experiment was performed as previously described[39]. Briefly, sections (4 $\mu \mathrm{m}$ thick) of paraffinembedded samples were deparaffinized and rehydrated in a graded series of ethanol. After inactivation of endogenous peroxidase activity with $3 \% \mathrm{H}_{2} \mathrm{O}_{2}$ in methanol for $10 \mathrm{~min}$, the sections were washed three times in PBS and blocked with goat serum for $20 \mathrm{~min}$. Then, they were incubated with primary antibodies in a humid container at $4^{\circ} \mathrm{C}$ overnight. After the addition of the PowerVisionTM complex, tissue sections were incubated at $37^{\circ} \mathrm{C}$ for 20 min followed by treatment with DAB. PBS in place of primary antibody was used as a negative control.

\section{Statistical analysis}

The data are presented as the mean \pm s.e.m. from at least three independent experiments. Statistical analysis was performed using GraphPad Prism software. A random number table was used to randomize the mice into control and treatment groups. The numbers of mice were determined on the basis of our pretests and previous experience with similar experiments. A value of $P<0.05$ was considered statistically significant. The statistical tests were two-sided.

\section{Declarations}

\section{Acknowledgments}


We thank Ms. Wang Wei for drawing the Graphical Abstract.

\section{Authors' contributions}

X.L. and Q.Y. contributed equally to this work. Y.G., C.Z. and Y.Z. contributed to project conceptualization and supervision. Y.G., X.L., Q.Y., C.L., X.G., R.Z. and K.Z. contributed to the investigation. W.Z., S.W., Q.H., W.L. and M.L. contributed to the data curation, Y.G. and X.L. contributed to writing the original draft, Y.G., C.Z., Y.Z. and X.L. contributed to writing, reviewing, and editing the manuscript, and Y.G., C.Z. and Y.Z. contributed to funding acquisition.

\section{Funding}

This work was financially supported by grants from the Hong Kong Scholar Program, the National Natural Science Foundation of China (NSFC), NO. 81902678, 81802632, 82072910, and the Key Research and Development Program of Shaanxi Province (NO. 2017ZDCXL-SF-01-03).

\section{Availability of data and materials}

The supplementary file was uploaded with the manuscript.

\section{Declarations}

\section{Ethics approval and consent to participate}

The animal experiments were performed in accordance with a protocol approved by the Institutional Animal Care and Use Committee of Air Force Medical University.

\section{Consent for publication}

The corresponding author of this manuscript, Yuan Gao, Cun Zhang and Yingqi Zhang on behalf of all coauthors (Xiaoju Li, Qianqian Yu, Xinyan Guo, Chenlin Liu, Runze Zhao, Kuo Zhang, Wangqian Zhang, Jinghan Liu, Shuning Wang, Qiang Hao, Weina Li and Meng Li) declares the consent of publication of this manuscript in the Journal of Nanobiotechnology. All authors have read and approved the submitted final version.

\section{Competing interests}

The authors declare no conflicts of interest.

\section{References}

1. Siegel RL, Miller KD, Jemal A. Cancer statistics, 2018. CA Cancer J Clin. 2018, 68: 7-30. 
2. Bray F, Ferlay J, Soerjomataram I, Siegel RL, Torre LA, Jemal A. Global cancer statistics 2018: GLOBOCAN estimates of incidence and mortality worldwide for 36 cancers in 185 countries. CA Cancer J Clin. 2018, 68: 394-424.

3. Yang JD, Hainaut P, Gores GJ, Amadou A, Plymoth A, Roberts LR. A global view of hepatocellular carcinoma: trends, risk, prevention and management. Nat Rev Gastroenterol Hepatol. 2019, 16: 589604.

4. Cheng AL, Kang YK, Chen Z, Tsao CJ, Qin S, Kim JS, et al. Efficacy and safety of sorafenib in patients in the Asia-Pacific region with advanced hepatocellular carcinoma: a phase III randomised, doubleblind, placebo-controlled trial. Lancet Oncol. 2009, 10: 25-34.

5. Chen X, Kang R, Kroemer G, Tang D. Broadening horizons: the role of ferroptosis in cancer. Nat Rev Clin Oncol. 2021, 18: 280-96.

6. Wang Q, Bin C, Xue Q, Gao Q, Huang A, Wang K, et al. GSTZ1 sensitizes hepatocellular carcinoma cells to sorafenib-induced ferroptosis via inhibition of NRF2/GPX4 axis. Cell Death Dis. 2021, 12: 426.

7. Su Y, Zhao B, Zhou L, Zhang Z, Shen Y, Lv H, et al. Ferroptosis, a novel pharmacological mechanism of anti-cancer drugs. Cancer Lett. 2020, 483: 127-36.

8. Sun X, Ou Z, Chen R, Niu X, Chen D, Kang R, et al. Activation of the p62-Keap1-NRF2 pathway protects against ferroptosis in hepatocellular carcinoma cells. Hepatology. 2016, 63: 173-84.

9. Lachaier E, Louandre C, Godin C, Saidak Z, Baert M, Diouf M, et al. Sorafenib induces ferroptosis in human cancer cell lines originating from different solid tumors. Anticancer Res. 2014, 34: 6417-22.

10. Dixon SJ, Patel DN, Welsch M, Skouta R, Lee ED, Hayano M, et al. Pharmacological inhibition of cystine-glutamate exchange induces endoplasmic reticulum stress and ferroptosis. Elife. 2014, 3 : e2523.

11. Coriat R, Nicco C, Chereau C, Mir O, Alexandre J, Ropert S, et al. Sorafenib-induced hepatocellular carcinoma cell death depends on reactive oxygen species production in vitro and in vivo. Mol Cancer Ther. 2012, 11: 2284-93.

12. Dixon SJ, Stockwell BR. The role of iron and reactive oxygen species in cell death. Nat Chem Biol. 2014, 10: 9-17.

13. Seibt TM, Proneth B, Conrad M. Role of GPX4 in ferroptosis and its pharmacological implication. Free Radic Biol Med. 2019, 133: 144-52.

14. Feng $\mathrm{H}$, Stockwell BR. Unsolved mysteries: How does lipid peroxidation cause ferroptosis? Plos Biol. 2018, 16: e2006203.

15. Wang F, Min J. DHODH tangoing with GPX4 on the ferroptotic stage. Signal Transduct Target Ther. 2021, 6: 244.

16. Herrmann IK, Wood M, Fuhrmann G. Extracellular vesicles as a next-generation drug delivery platform. Nat Nanotechnol. 2021, 16: 748-59. 
17. Wozniak AL, Adams A, King KE, Dunn W, Christenson LK, Hung WT, et al. The RNA binding protein FMR1 controls selective exosomal miRNA cargo loading during inflammation. J Cell Biol. 2020, 219.

18. Bolduc F, Turcotte MA, Perreault JP. The Small Nuclear Ribonucleoprotein Polypeptide A (SNRPA) binds to the G-quadruplex of the BAG-1 5'UTR. Biochimie. 2020, 176: 122-7.

19. Zhou T, Liang X, Wang P, Hu Y, Qi Y, Jin Y, et al. A Hepatocellular Carcinoma Targeting Nanostrategy with Hypoxia-Ameliorating and Photothermal Abilities that, Combined with Immunotherapy, Inhibits Metastasis and Recurrence. Acs Nano. 2020, 14: 12679-96.

20. Garcia-Bermudez J, Birsoy K. A mitochondrial gatekeeper that helps cells escape death by ferroptosis. Nature. 2021, 593: 514-5.

21. Stockwell BR, Friedmann AJ, Bayir H, Bush Al, Conrad M, Dixon SJ, et al. Ferroptosis: A Regulated Cell Death Nexus Linking Metabolism, Redox Biology, and Disease. Cell. 2017, 171: 273-85.

22. Zhang Y, Shi J, Liu X, Feng L, Gong Z, Koppula P, et al. BAP1 links metabolic regulation of ferroptosis to tumour suppression. Nat Cell Biol. 2018, 20: 1181-92.

23. Mao C, Liu X, Zhang Y, Lei G, Yan Y, Lee H, et al. DHODH-mediated ferroptosis defence is a targetable vulnerability in cancer. Nature. 2021, 593: 586-90.

24. Fu Z, Zhang X, Zhou X, Ur-Rehman U, Yu M, Liang H, et al. In vivo self-assembled small RNAs as a new generation of RNAi therapeutics. Cell Res. 2021, 31: 631-48.

25. van Niel G, D'Angelo G, Raposo G. Shedding light on the cell biology of extracellular vesicles. Nat Rev Mol Cell Biol. 2018, 19: 213-28.

26. Ohno S, Takanashi M, Sudo K, Ueda S, Ishikawa A, Matsuyama N, et al. Systemically injected exosomes targeted to EGFR deliver antitumor microRNA to breast cancer cells. Mol Ther. 2013, 21: $185-91$.

27. Kooijmans S, Stremersch S, Braeckmans K, de Smedt SC, Hendrix A, Wood M, et al. Electroporationinduced siRNA precipitation obscures the efficiency of siRNA loading into extracellular vesicles. $J$ Control Release. 2013, 172: 229-38.

28. Gao Y, Li X, Zeng C, Liu C, Hao Q, Li W, et al. CD63(+) Cancer-Associated Fibroblasts Confer Tamoxifen Resistance to Breast Cancer Cells through Exosomal miR-22. Adv Sci (Weinh). 2020, 7: 2002518.

29. Zhang $H$, Deng $T$, Liu R, Ning T, Yang H, Liu D, et al. CAF secreted miR-522 suppresses ferroptosis and promotes acquired chemo-resistance in gastric cancer. Mol Cancer. 2020, 19: 43.

30. Yu M, Gai C, Li Z, Ding D, Zheng J, Zhang W, et al. Targeted exosome-encapsulated erastin induced ferroptosis in triple negative breast cancer cells. Cancer Sci. 2019, 110: 3173-82.

31. Lu B, Chen XB, Ying MD, He QJ, Cao J, Yang B. The Role of Ferroptosis in Cancer Development and Treatment Response. Front Pharmacol. 2017, 8: 992.

32. Stockwell BR, Jiang X. The Chemistry and Biology of Ferroptosis. Cell Chem Biol. 2020, 27: 365-75.

33. Wu Y, Yu C, Luo M, Cen C, Qiu J, Zhang S, et al. Ferroptosis in Cancer Treatment: Another Way to Rome. Front Oncol. 2020, 10: 571127. 
34. Yi J, Minikes AM, Jiang X. Aiming at Cancer In Vivo: Ferroptosis-Inducer Delivered by Nanoparticles. Cell Chem Biol. 2019, 26: 621-2.

35. Terasawa $K$, Tomabechi $Y$, Ikeda $M$, Ehara $H$, Kukimoto-Niino $M$, Wakiyama $M$, et al. Lysosomeassociated membrane proteins- 1 and -2 (LAMP-1 and LAMP-2) assemble via distinct modes. Biochem Biophys Res Commun. 2016, 479: 489-95.

36. Armstrong JP, Holme MN, Stevens MM. Re-Engineering Extracellular Vesicles as Smart Nanoscale Therapeutics. Acs Nano. 2017, 11: 69-83.

37. Tian Y, Li S, Song J, Ji T, Zhu M, Anderson GJ, et al. A doxorubicin delivery platform using engineered natural membrane vesicle exosomes for targeted tumor therapy. Biomaterials. 2014, 35: 2383-90.

38. Gao Y, Wang Z, Hao Q, Li W, Xu Y, Zhang J, et al. Loss of ERalpha induces amoeboid-like migration of breast cancer cells by downregulating vinculin. Nat Commun. 2017, 8: 14483.

\section{Figures}


A

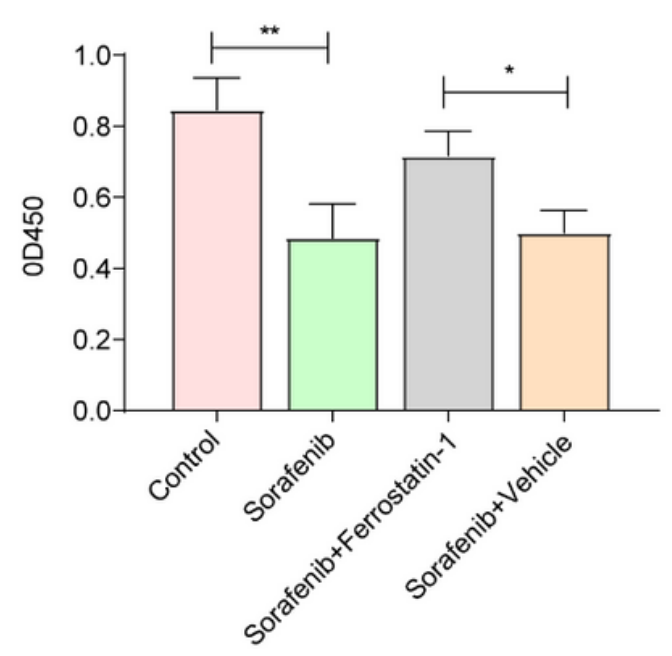

D

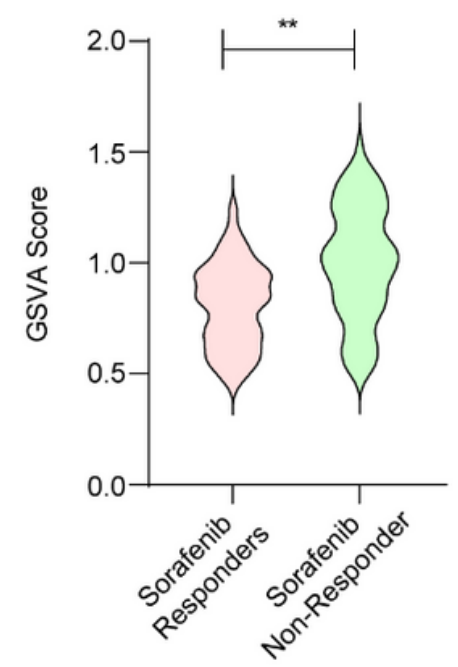

B
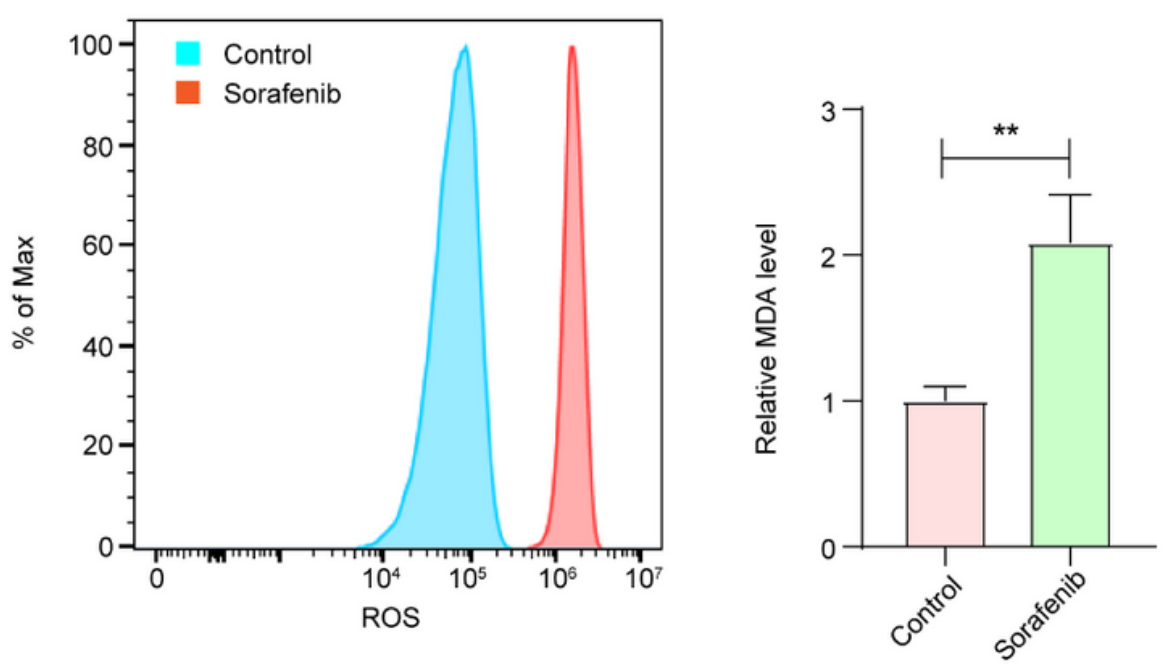

E

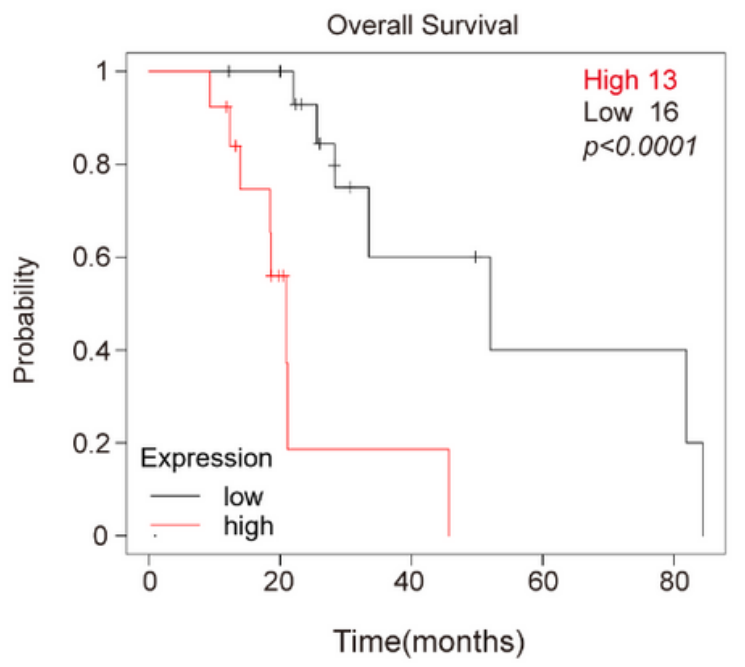

\section{Figure 1}

Reduced ferroptotic activity during sorafenib treatment is associated with compromised therapeutic efficiency. (A) Viability of HepG-2 cells as measured by the CCK-8 assay. Ferrostatin-1 is a ferroptosis inhibitor. Sorafenib $(10 \mu \mathrm{M})$. (B) HepG-2 cells were treated with $10 \mu \mathrm{M}$ sorafenib, and ROS production was assessed by DCFH-DA staining followed by flow cytometry. (C) MDA levels in HepG-2 cells treated with vehicle or $10 \mu \mathrm{M}$ sorafenib for $24 \mathrm{~h}$. (D) GSVA was conducted to calculate the score for enrichment of ferroptosis suppressor genes. HCC tissues from sorafenib responders or sorafenib nonresponders were obtained from the Gene Expression Omnibus (GSE1109211). (E) Kaplan-Meier analysis of overall survival based on the expression signature of ferroptosis suppressor genes. (A, C) The data are shown as the means \pm S.E.M. (A) ANOVA with Dunnett's t-test. (C, D) Unpaired t-test. (E) Log-rank test. ${ }^{*}<<0.05$, ${ }^{*} p$ $<0.01$. 
A

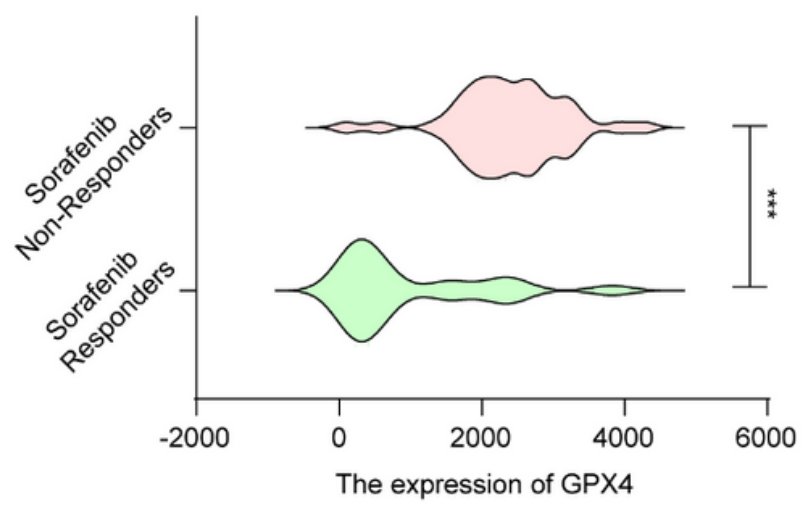

B

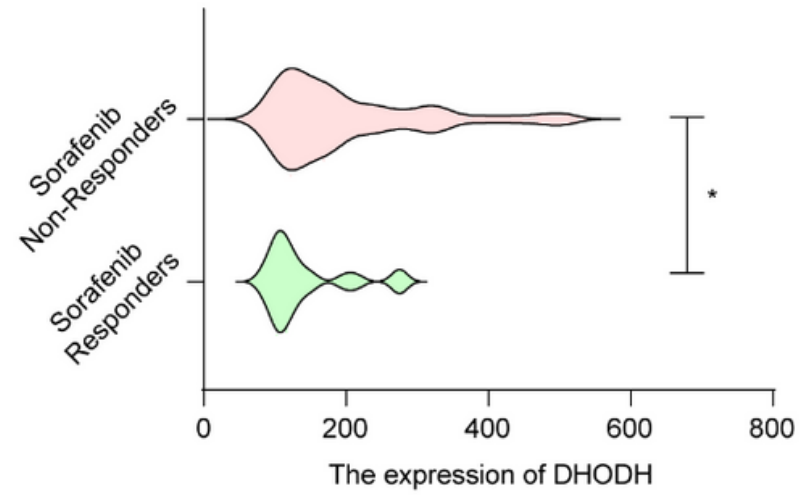

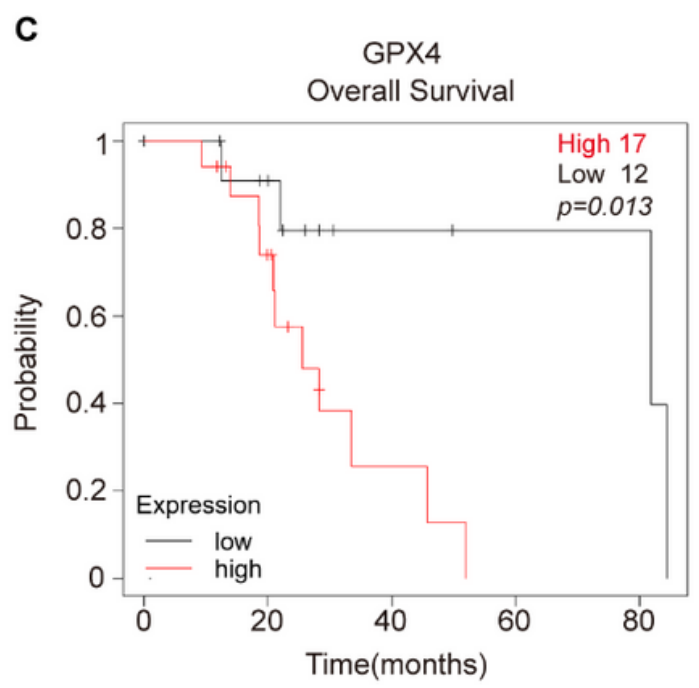

$\mathbf{F}$
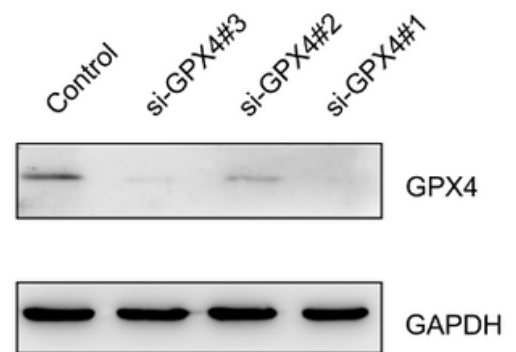

D

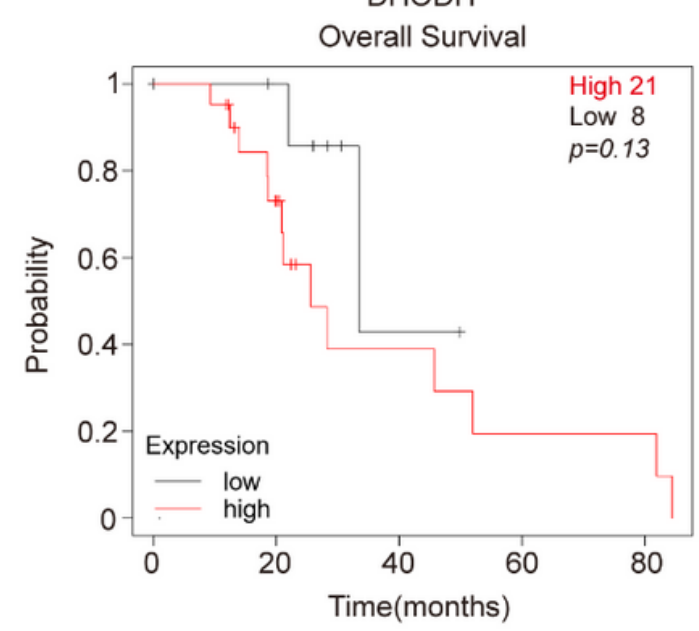

E

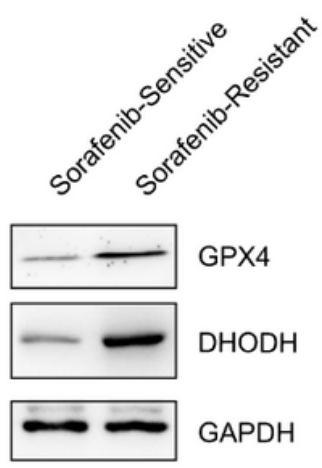

G

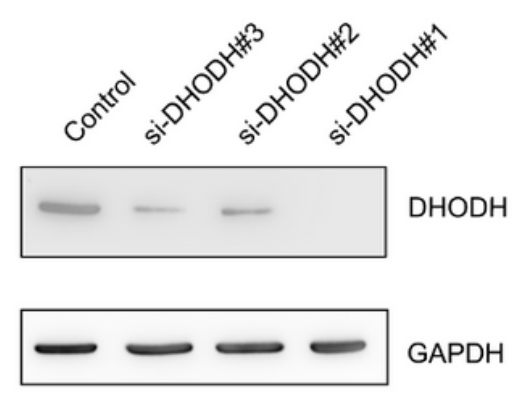

H

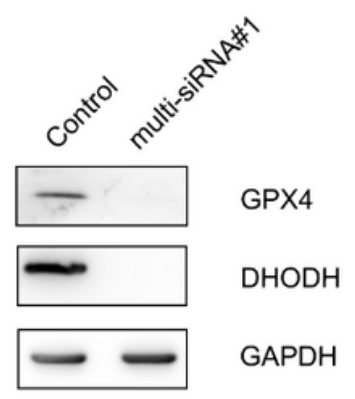

\section{Figure 2}

Gene-silencing activities of multi-siRNA against GPX4 and DHODH genes. (A) GPX4 expression in HCC tissues from sorafenib responders $(n=21)$ or sorafenib nonresponders $(n=43)$ (gse109211). (B) That of DHODH in the HCC tissues of sorafenib responders $(n=21)$ or sorafenib nonresponders $(n=43)$ (gse109211). (C) Kaplan-Meier overall survival analysis of the GPX4 gene in HCC patients who received sorafenib treatment. (D) Kaplan-Meier overall survival analysis of the DHODH gene in HCC patients who received sorafenib treatment. (E) Western blotting for assessment of the protein levels of DHODH and GPX4 in sorafenib-sensitive HepG-2 or sorafenib-resistant HepG-2 cells. (F) Western blotting was 
conducted to detect GPX4 expression in HepG-2 cells transfected with scramble or GPX4 siRNAs. (G) Western blotting was conducted to detect DHODH expression in HepG-2 cells transfected with scramble or DHODH siRNAs. $(\mathrm{H})$ HepG-2 cells were transfected with scramble siRNA or multi-siRNA\#1 containing the si-GPX4\#1 and si-DHODH\#1 target sequences, and Western blotting was conducted to detect GPX4 and DHODH expression. (A, B) Unpaired t-test. (C, D) Log-rank test. ${ }^{*}<<0.05,{ }^{* \star} p<<0.001$.

A

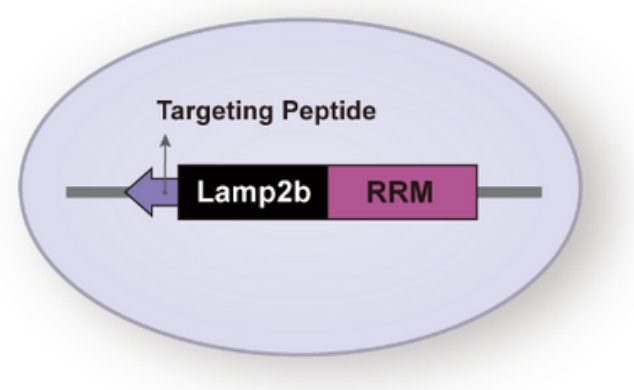

C

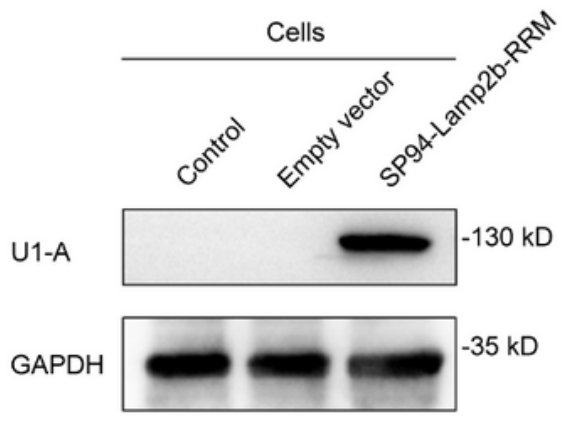

E
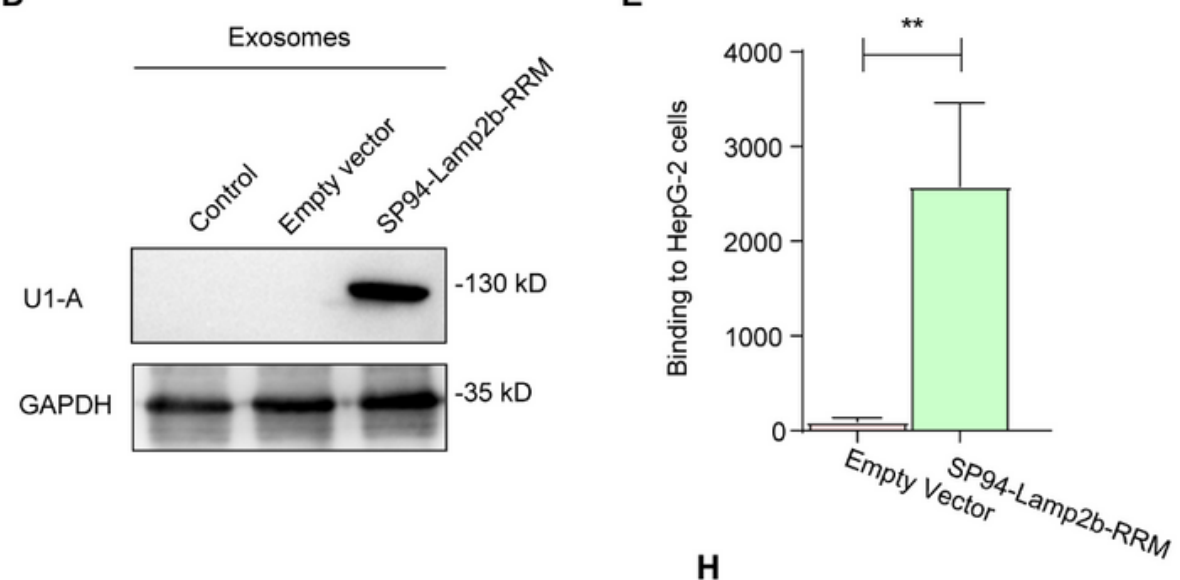

G

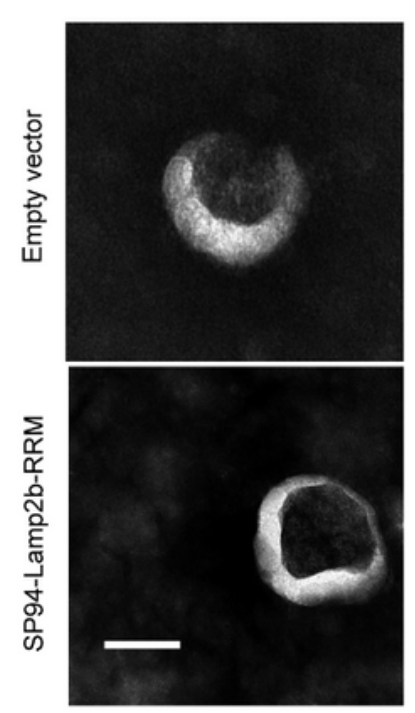

Figure 3 
Preparation and characterization of SP94-Lamp2b-RRM fusion protein-engineered exosomes (A) Schematic representation of the hepatoma-cell-targeting peptide SP94 and the RRM of U1-A fused to the $\mathrm{N}$-terminus and C-terminus of Lamp2b. (B) Schematic representation of the structure of the SP94Lamp2b-RRM fusion protein and the engineered exosomes. (C) Western blot analysis of U1-A expression in HEK-293T cells transfected with empty vector or SP94-Lamp2b-RRM plasmids. (D) U1-A expression in exosomes derived from HEK-293T cells transfected with empty vector or SP94-Lamp2b-RRM plasmids. (E) Binding of DiO-labeled blank exosomes or SP94-Lamp2b-RRM exosomes to immobilized HepG-2 cells was assessed by measuring the fluorescence of the wells after they were washed to remove unbound exosomes. Exosomes were incubated with immobilized HepG-2 cells for $1 \mathrm{~h}$. (F) Western blot analysis of the expression of the exosome markers CD63, CD9 and TSG101 on exosomes derived from HEK-293T cells transfected with different plasmids. (G) Nanoparticle tracking analysis shows the size distribution of exosomes derived from HEK-293T cells transfected with different plasmids. (H) Representative TEM images of exosomes derived from control or SP94-Lamp2b-RRM plasmid-transfected HEK-293T cells (scale bar, $100 \mathrm{~nm}$ ). (E) Unpaired t-test. **p $<0.01$. 
A

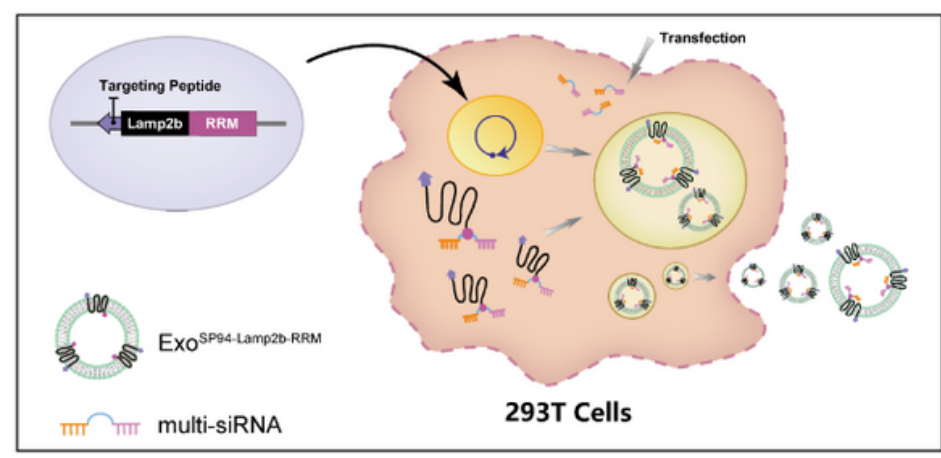

C

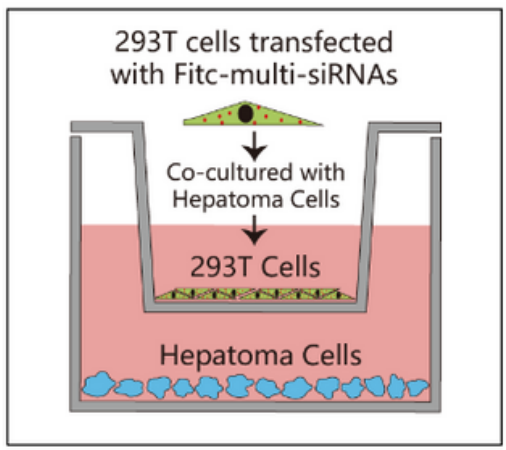

E

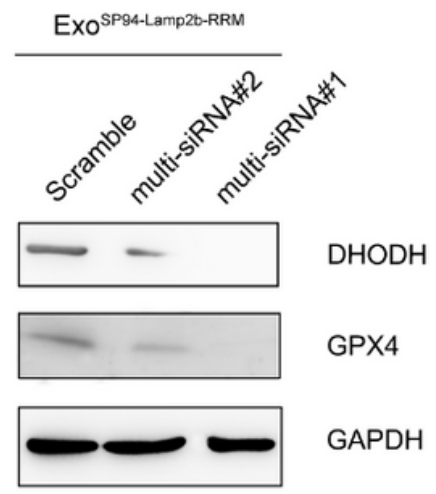

B

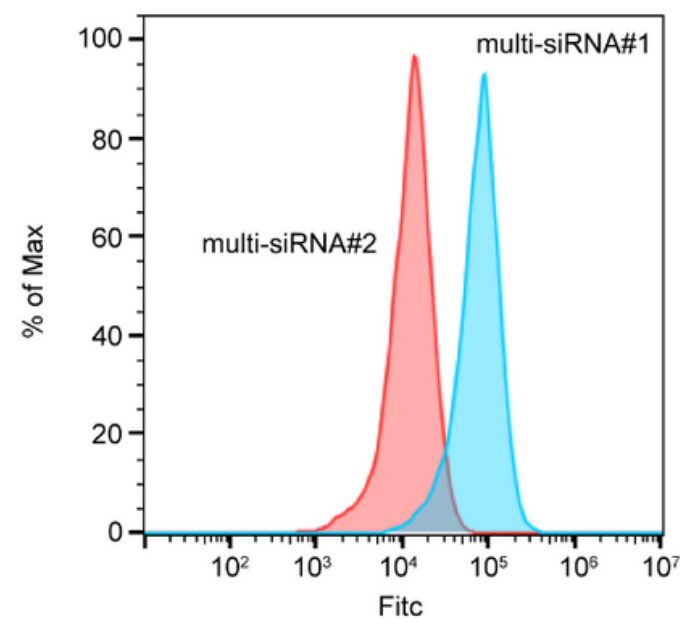

D
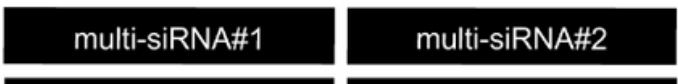
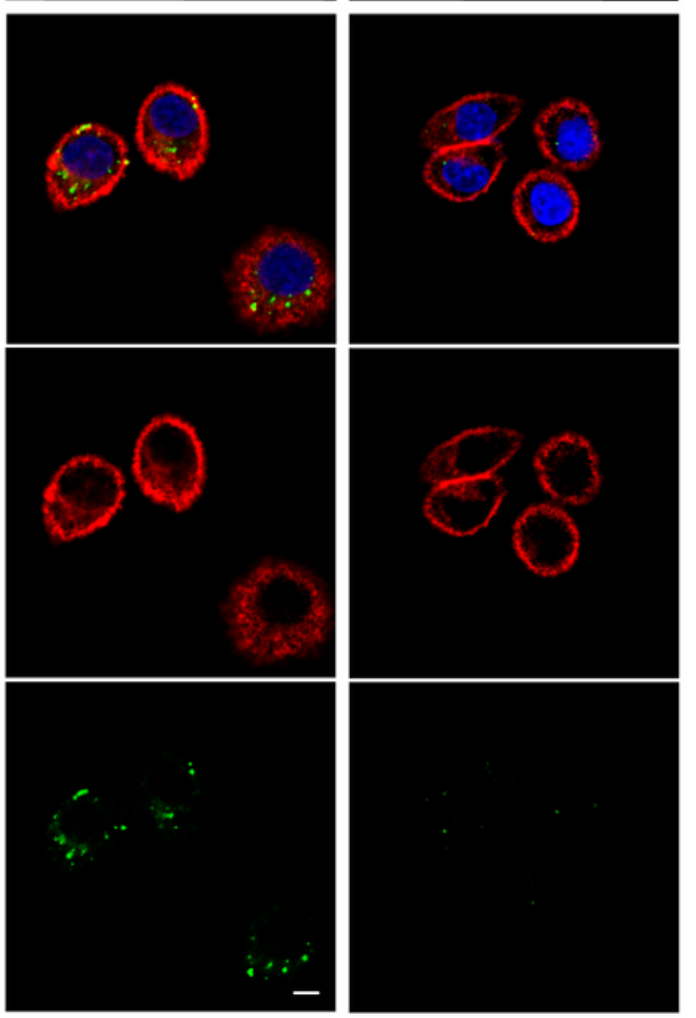

\section{Figure 4}

Efficient therapeutic cargo encapsulation into engineered exosomes expressing SP94-Lamp2b-RRM fusion protein. (A) Schematic diagram of the process of multi-siRNA encapsulation into exosomes via the SP94-Lamp2b-RRM fusion protein in HEK-293T cells. The SP94-Lamp2b-RRM fusion protein recruits multi-siRNA containing the sequence "AUUGCAC" to exosomes via RNA-RRM recognition. (B) HEK-293T cells transiently transfected with FITC-tagged multi-siRNA\#1 or FITC-tagged multi-siRNA\#2. Then, exosomes were collected for the flow cytometry assay. (C-D) HEK-293T cells transiently transfected with 
FITC-tagged multi-siRNAs were cocultured with HepG-2 cells for $24 \mathrm{~h}$ (multi-siRNA\#1, contains si-GPX4\#1, si-DHODH\#1 and the RRM binding sequence, multi-siRNA\#2, contains si-GPX4\#1 and si-DHODH\#1 without the RRM binding sequence). (C) Schematic illustration of HepG-2 and HEK-293T cell coculturing. (D) Representative confocal images of FITC (green) and F-actin (red) staining in HepG-2 cells. The nuclei were counterstained with DAPI (blue) (scale bar, $10 \mu \mathrm{m}$ ). (E) HepG-2 cells were treated with functionalized exosomes derived from HEK-293 T cells and containing SP94-Lamp2b-RRM, and Western blot assays were conducted to detect GPX4 and DHODH expression in HepG-2 cells. Scramble: 1*106 HEK-293T cells were treated with the same amount of scramble siRNA, multi-siRNA\#2: 1*106 HEK-293T cells were treated with the same amount of multi-siRNA\#2, multi-siRNA\#1: 1*106 HEK-293T cells were treated with the same amount of multi-siRNA\#1. After $24 \mathrm{~h}$, the exosomes were collected and added to HepG-2 cells. 
A

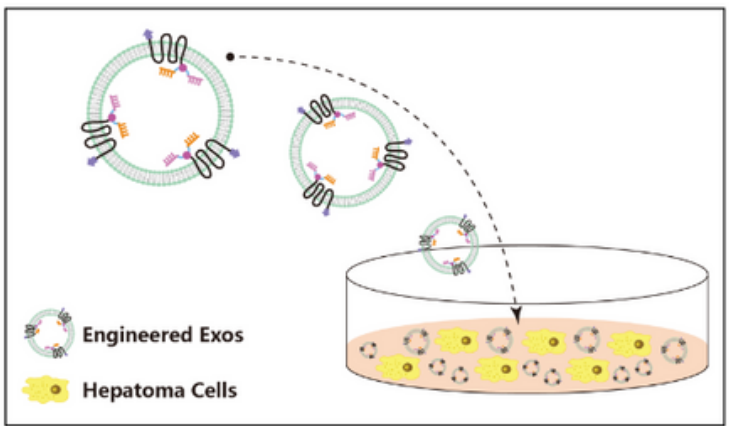

C

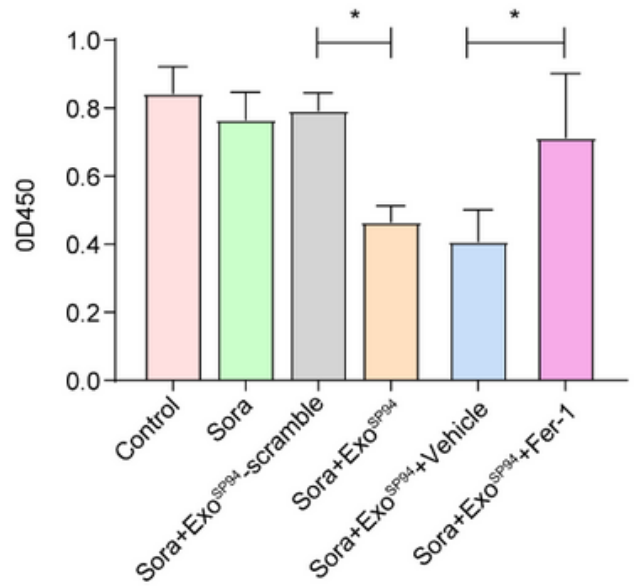

E

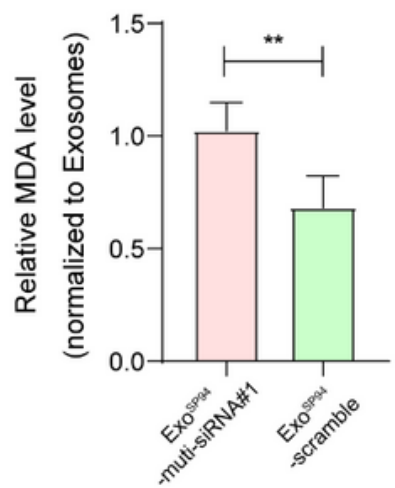

B

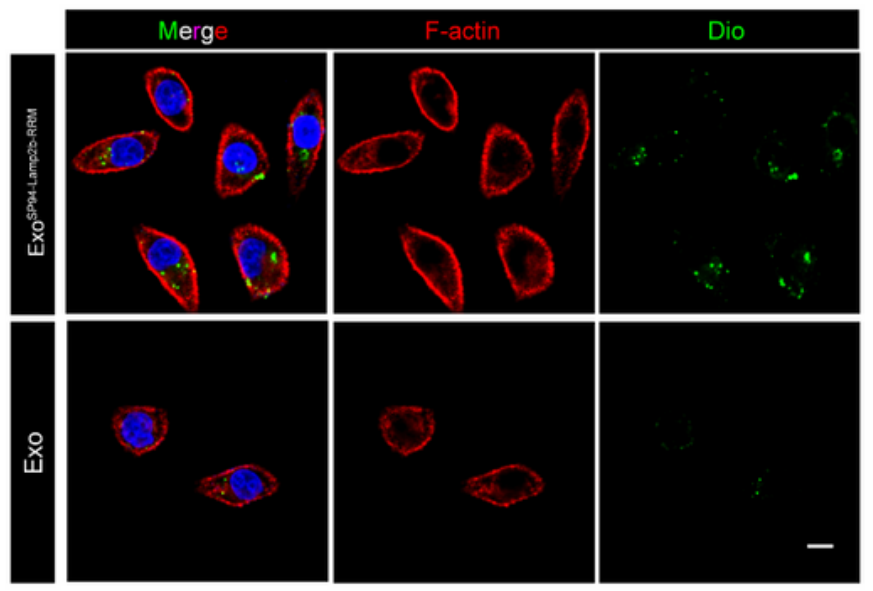

D

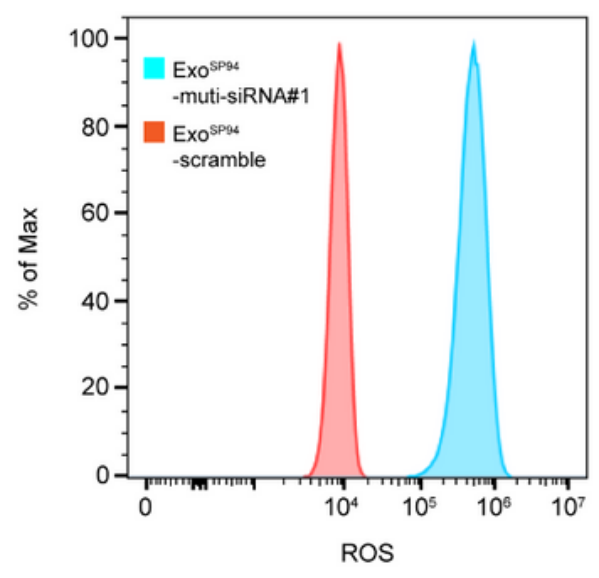

$\mathbf{F}$

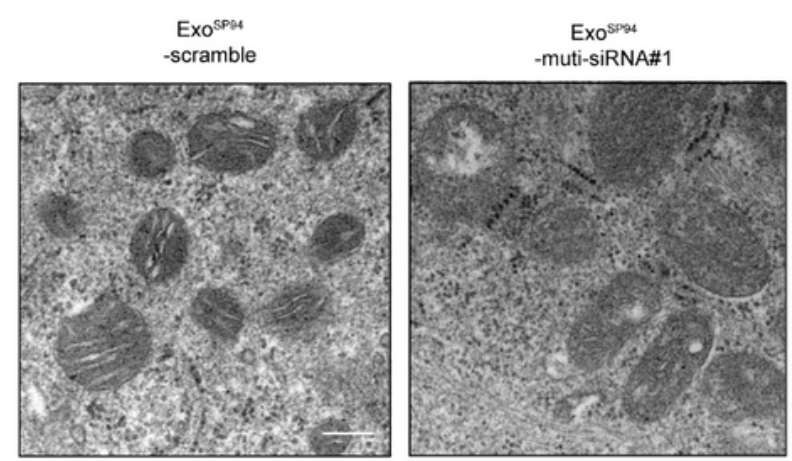

\section{Figure 5}

SP94-Lamp2b-RRM-functionalized exosomes could overcome sorafenib resistance by enhancing sorafenib-induced ferroptosis in HCC cells. (A) Illustration of the procedure by which HCC cells were treated with SP94-Lamp2b-RRM-functionalized exosomes encapsulating the multi-siRNA. (B) Control or SP94-Lamp2b-RRM-functionalized exosomes derived from HEK-293T cells were dyed with DiO, and exosome endocytosis was analyzed by confocal microscopy. The nuclei and F-actin were counterstained 
with DAPI (blue) and TRITC phalloidin (red), respectively (scale bar, $10 \mu \mathrm{m}$ ). (C) Viability of sorafenibresistant HepG-2 cells as measured by the CCK-8 assay. Sora: sorafenib treatment, ExoSP94-scramble: SP94-Lamp2b-RRM-functionalized exosomes containing scramble multi-RNA, ExoSP94: SP94-Lamp2bRRM-functionalized exosomes containing multi-RNA\#1, Fer-1: Ferrostatin-1 (D) Sorafenib-resistant HepG2 cells were treated with sorafenib in combination with SP94-Lamp2b-RRM-functionalized exosomes containing scramble or multi-siRNA\#1, and the ROS production was assessed by DCFH-DA staining followed by flow cytometry. (E) MDA levels of sorafenib-resistant HepG-2 cells treated with sorafenib and SP94-Lamp2b-RRM-functionalized exosomes containing scramble or multi-siRNA\#1. (F) TEM images of mitochondria in sorafenib-resistant HepG-2 cells cotreated with sorafenib and SP94-Lamp2b-RRMfunctionalized exosomes containing scramble or multi-siRNA\#1. (C, E) The data are shown as the means \pm S.E.M. (C) ANOVA with Dunnett's t-test. (E) Unpaired t-test. ${ }^{*} p<0.05,{ }^{\star *} p<0.01$. 
A

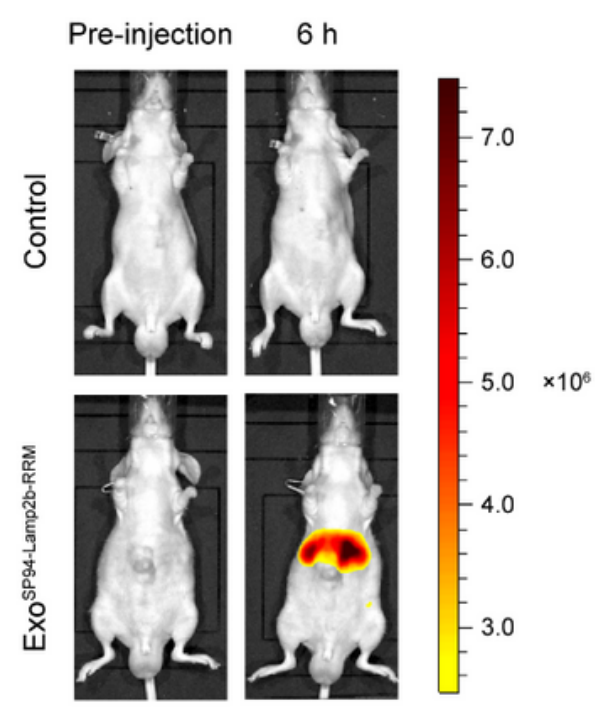

C

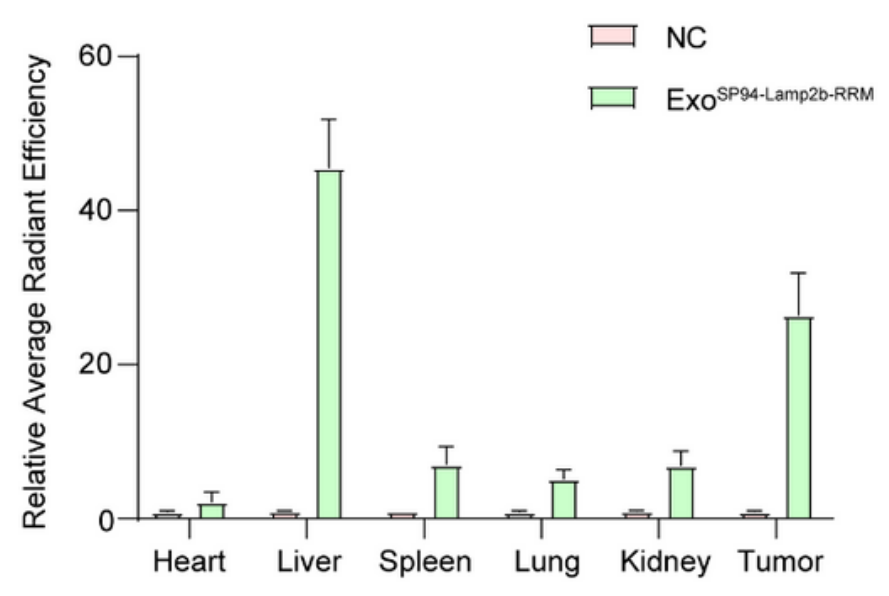

B

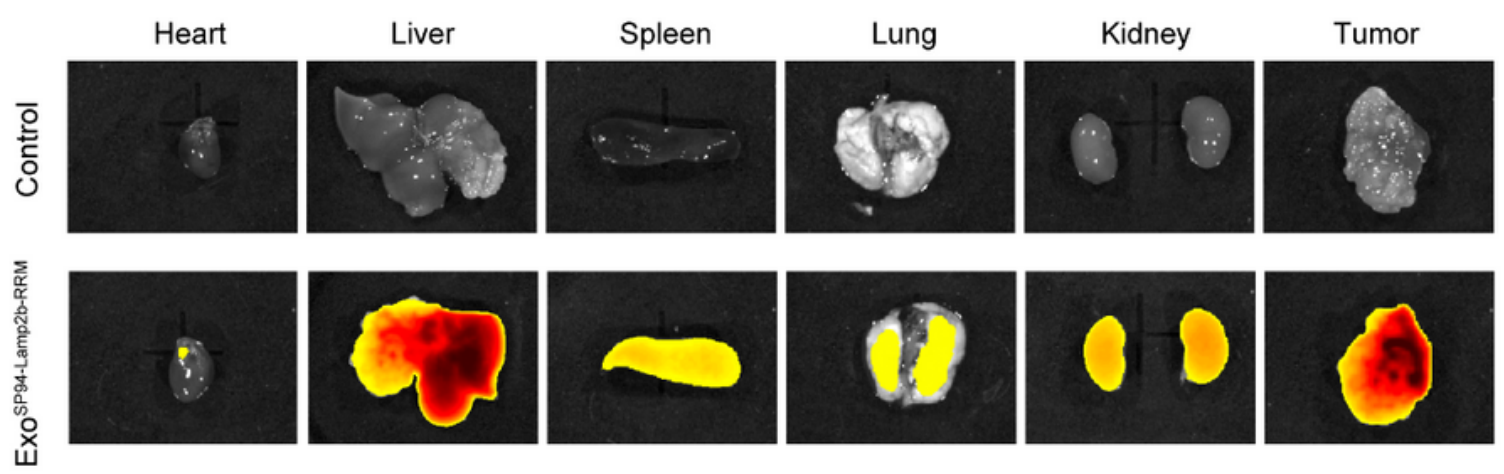

D

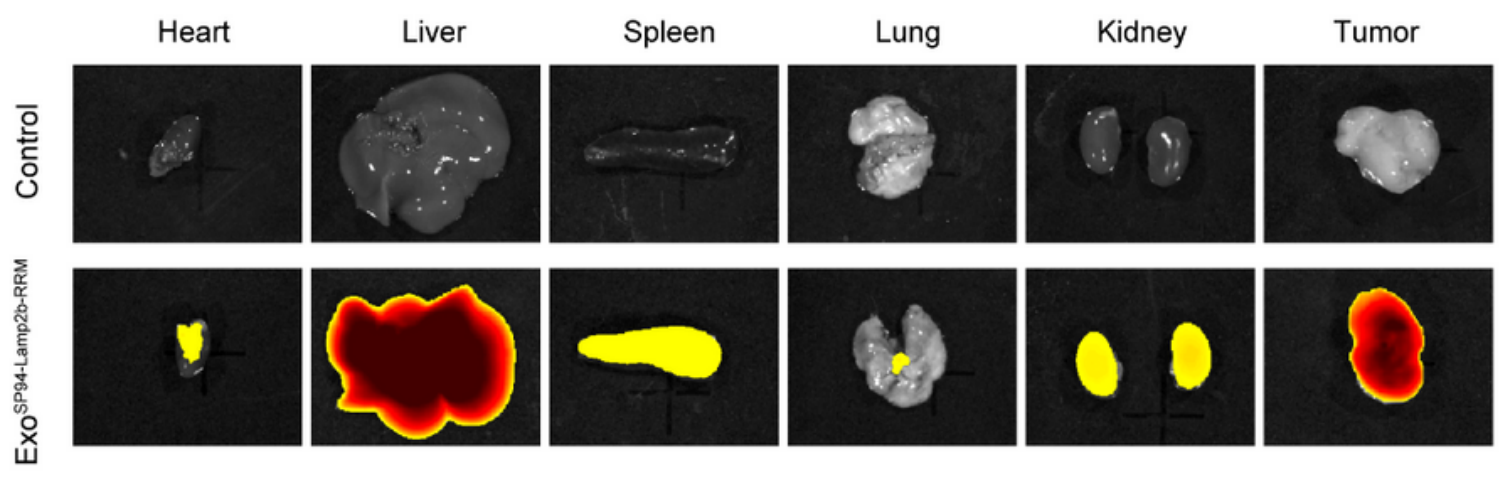

\section{Figure 6}

SP94-Lamp2b-RRM-functionalized exosomes could efficiently target HCC in vivo. (A-C) Sorafenibresistant HepG-2 cells were orthotopically inoculated into the left liver lobes of nude mice. SP94-Lamp2bRRM-functionalized exosomes $(100 \mu \mathrm{g})$ were injected into nude mice via tail vein. (A) Mice were evaluated with a fluorescent live imaging system $6 \mathrm{~h}$ after injection. (B) Representative ex vivo fluorescence images of DiR-labeled exosomes in the main organs and HCC tissues. (C) Relative 
fluorescence intensity of DiR-labeled exosomes in different organs and liver cancer tissues. (D) Sorafenibresistant HepG-2 cells were subcutaneously inoculated on the left backs of mice. SP94-Lamp2b-RRMfunctionalized exosomes were loaded with $\mathrm{DiR}$, and $100 \mathrm{\mu g}$ of exosomes was injected into mice via tail vein. Different organs and tumors were harvested to conduct fluorescence imaging. (C) The data are shown as the means \pm S.E. $M, n=5$.

A

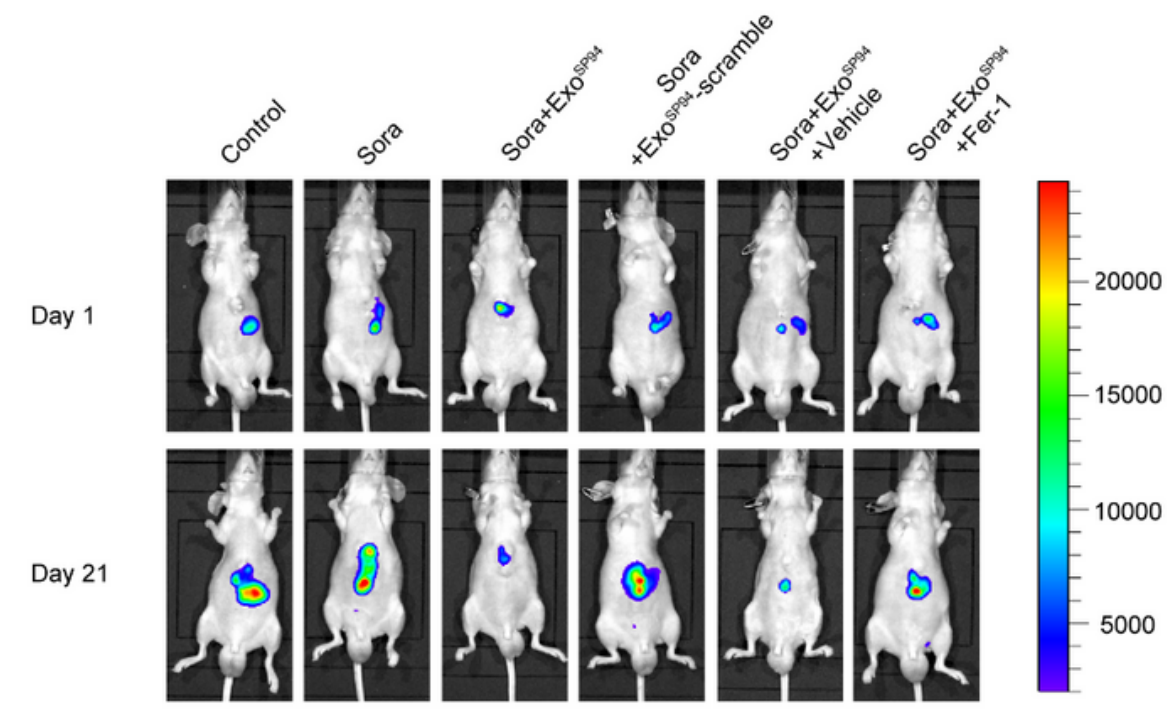

B

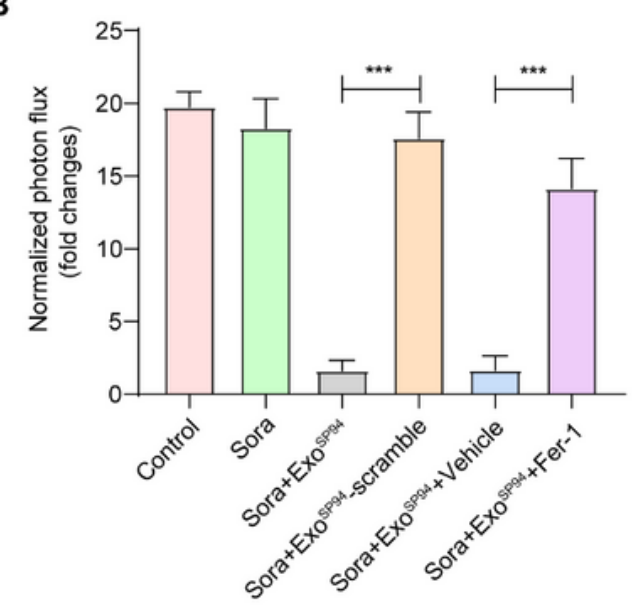

C

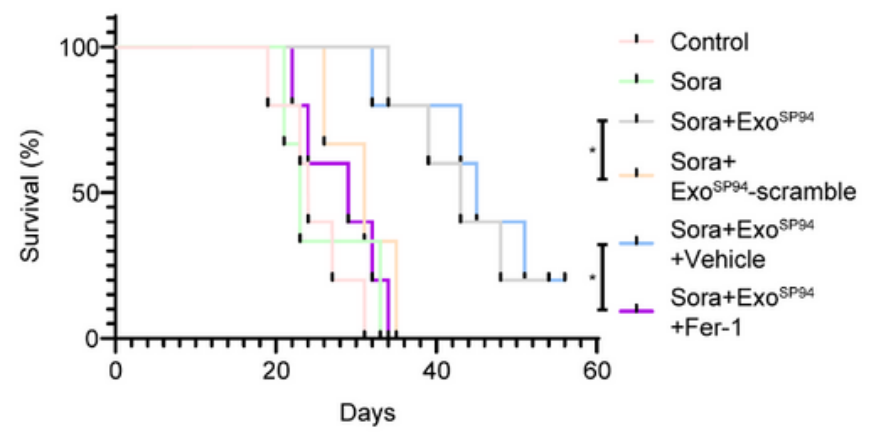

D

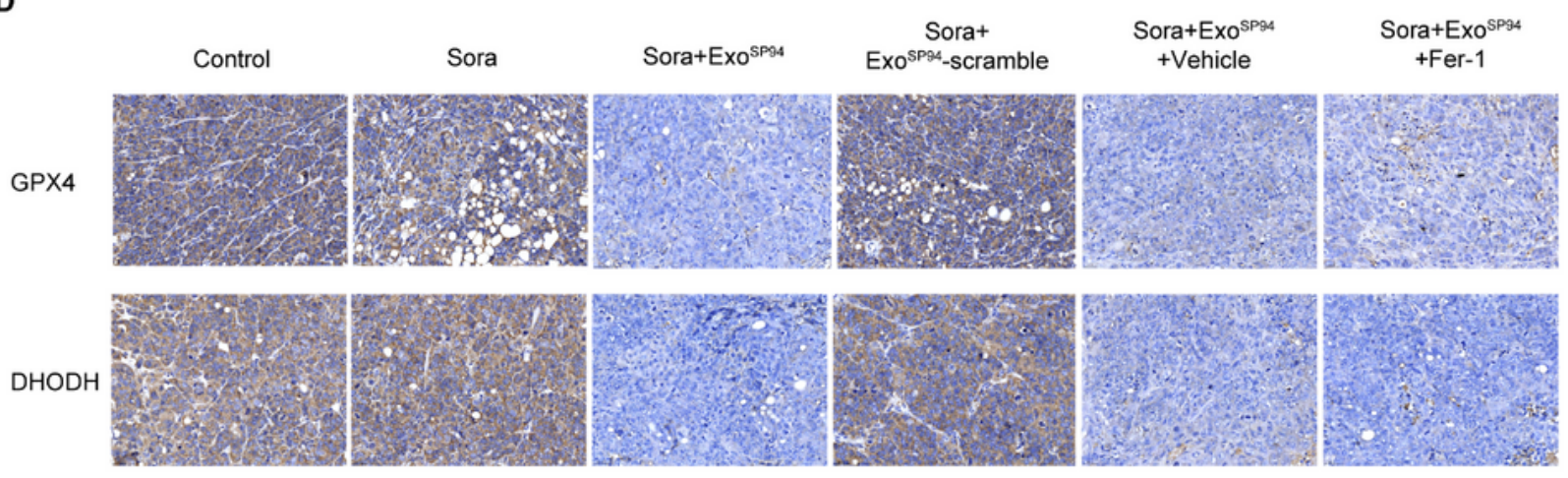

Figure 7 
Therapeutic effects of SP94-Lamp2b-RRM-functionalized exosomes and sorafenib in an HCC mouse model. Sorafenib-resistant HepG-2 cells were orthotopically injected into the left liver lobes of nude mice. Mice with a similar tumor size were used. Control: saline, Sora: sorafenib, Sora+ExoSP94: sorafenib plus SP94-Lamp2b-RRM-functionalized exosomes containing multi-siRNA\#1, Sora+ExoSP94-scramble: sorafenib plus SP94-Lamp2b-RRM-functionalized exosomes containing scramble siRNA, Sora+ExoSP94+Vehicle: sorafenib plus SP94-Lamp2b-RRM-functionalized exosomes containing multisiRNA\#1 in vehicle, Sora+ExoSP94+Fer-1: sorafenib plus SP94-Lamp2b-RRM-functionalized exosomes in multi-siRNA\#1 combined with ferrostatin-1. Tumor growth was measured with an IVIS imaging system. (A) Representative tumor imaging on days 1 and 21 after the first treatment. (B) The normalized photon flux at day 21 was analyzed ( $n=5)$. (C) Survival of mice from different groups $(n=5)$. (D) Representative immunohistochemical staining for GPX4 and DHODH in HCC tissues from different groups (scale bar, 25 $\mu \mathrm{m})$. (B) The data are shown as the means \pm S.E.M. ANOVA with Dunnett's t-test. (C) Log-rank test. ***p $<0.001$.

A

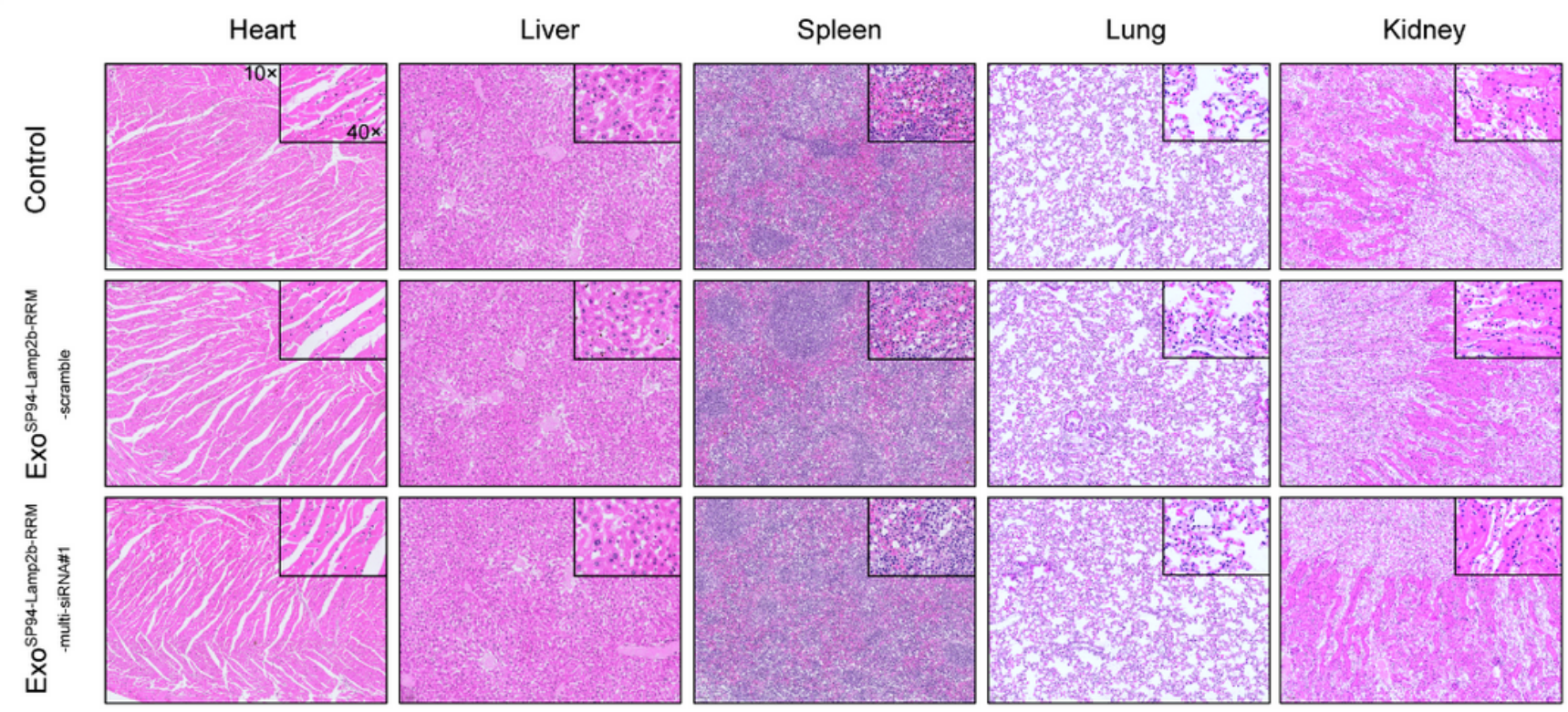

\section{Figure 8}

Systemic toxicity evaluation. One hundred micrograms of SP94-Lamp2b-RRM-functionalized exosomes were injected into nude mice via tail vein every 3 days for 21 days. Control: saline, Sora+ExoSP94Lamp2b-RRM-scramble: sorafenib plus SP94-Lamp2b-RRM-functionalized exosomes containing scramble siRNA, Sora+ExoSP94-Lamp2b-RRM-multi-siRNA\#1: sorafenib plus SP94-Lamp2b-RRMfunctionalized exosomes containing multi-siRNA\#1, (A) Representative hematoxylin-eosin (H\&E) staining of the main organs from different treatment groups (scale bar, $100 \mu \mathrm{m}$ ).

\section{Supplementary Files}


This is a list of supplementary files associated with this preprint. Click to download.

- SupportingInformation.docx

- AG.png 